\title{
Genetic ancestry and the relationship of cigarette smoking to lung function and per cent emphysema in four race/ethnic groups: a cross-sectional study
}

\author{
Rhea Powell, ${ }^{1}$ Duncan Davidson, ${ }^{1}$ Jasmin Divers, ${ }^{2}$ Ani Manichaikul, ${ }^{3} \mathrm{~J}$ Jeffrey Carr, ${ }^{4}$ \\ Robert Detrano, ${ }^{5}$ Eric A Hoffman, ${ }^{6}$ Rui Jiang, ${ }^{1}$ Richard A Kronmal, ${ }^{7}$ Kiang Liu, ${ }^{8}$ \\ Naresh M Punjabi, ${ }^{9}$ Eyal Shahar, ${ }^{10}$ Karol E Watson, ${ }^{11}$ Jerome I Rotter, ${ }^{12}$ \\ Kent D Taylor, ${ }^{12}$ Stephen S Rich, ${ }^{3}$ R Graham Barr ${ }^{1,13}$
}

\begin{abstract}
- Additional material is published online only. To view please visit the journal online (http://dx.doi.org/10.1136/ thoraxjn-2012-202116).

For numbered affiliations see end of article.

Correspondence to Dr R Graham Barr, Department of Medicine, Columbia University Medical Center PH 9 East - Room 105, 630 West 168th St., New York, NY 10032, USA; rgb9@columbia.edu
\end{abstract}

RP and DD contributed equally.

Received 4 May 2012 Revised 7 March 2013 Accepted 12 March 2013 Published Online First 12 April 2013
To cite: Powell $R$, Davidson D, Divers J, et al. Thorax 2013;68:634-642.

\begin{abstract}
Background Cigarette smoking is the major cause of chronic obstructive pulmonary disease and emphysema. Recent studies suggest that susceptibility to cigarette smoke may vary by race/ethnicity; however, they were generally small and relied on self-reported race/ethnicity. Objective To test the hypothesis that relationships of smoking to lung function and per cent emphysema differ by genetic ancestry and self-reported race/ethnicity among Caucasians, African-Americans, Hispanics and Chinese-Americans.

Design Cross-sectional population-based study of adults age 45-84 years in the USA.
\end{abstract}

Measurements Principal components of genetic ancestry and continental ancestry estimated from one million genome-wide single nucleotide polymorphisms; pack-years of smoking; spirometry measured for 3344 participants; and per cent emphysema on computed tomography for 8224 participants.

Results The prevalence of ever-smoking was: Caucasians, 57.6\%; African-Americans, 56.4\%; Hispanics, 46.7\%; and Chinese-Americans, 26.8\%. Every 10 pack-years was associated with $-0.73 \%$ (95\% $\mathrm{Cl}-0.90 \%$ to $-0.56 \%)$ decrement in the forced expiratory volume in $1 \mathrm{~s}$ to forced vital capacity $\left(\mathrm{FEV}_{1}\right.$ to FVC) and a $0.23 \%(95 \% \mathrm{Cl} 0.08 \%$ to $0.38 \%)$ increase in per cent emphysema. There was no evidence that relationships of pack-years to the $\mathrm{FEV}_{1}$ to $\mathrm{FVC}$, airflow obstruction and per cent emphysema varied by genetic ancestry (all $p>0.10$ ), self-reported race/ethnicity (all $p>0.10)$ or, among African-Americans, African ancestry. There were small differences in relationships of packyears to the $\mathrm{FEV}_{1}$ among male Chinese-Americans and to the $F E V_{1}$ to $F V C$ ratio with African and Native American ancestry among male Hispanics only.

Conclusions In this large cohort, there was little to no evidence that the associations of smoking to lung function and per cent emphysema differed by genetic ancestry or self-reported race/ethnicity.

\section{INTRODUCTION}

Chronic obstructive pulmonary disease (COPD), defined by airflow obstruction that is not fully reversible, ${ }^{1}$ is anticipated to be the third leading cause of death worldwide by $2020 .^{2}$ Cigarette smoking is the primary cause of COPD and

\section{Key messages}

What is the key question?

- Does genetic ancestry or self-reported race and ethnicity modify the effect of cumulative packyears of smoking on lung function and emphysema?

What is the bottom line?

- The study identified no significant interaction between either genetic ancestry or self-reported race/ethnicity on the effect of smoking on lung function and emphysema in a large multiethnic cohort of adults in the United States.

\section{Why read on?}

- This study addresses a gap in knowledge about the understanding of differential risk for chronic obstructive pulmonary disease.

emphysema, ${ }^{3}$ characterised by destruction of alveolar walls and enlargement of air spaces distal to the terminal bronchioles, ${ }^{4}$ yet only some smokers develop these diseases. COPD outcomes vary by race/ethnic group. Mortality from COPD is the highest among Caucasians in the USA but is rising rapidly among African-Americans ${ }^{5}$ who have higher rates of hospitalisation and emergency department visits due to COPD. ${ }^{5}$ Further, COPD prevalence is higher among some Hispanic subgroups compared with African-Americans. ${ }^{6}$ It is unclear if differences in COPD outcomes result from variation in smoking patterns, healthcare access, environmental exposures or genetic susceptibility. The diverse US population provides an ideal setting to study differential effects of smoking on lung function.

A large meta-analysis suggested no difference in COPD risk for equivalent smoking history among African-Americans, Hispanics and Caucasians, but potentially a lower risk in Asian/Pacific Islanders, ${ }^{7}$ while other studies suggested that African-Americans are at an increased risk; ${ }^{8-11}$ yet another suggested decreased risk among Hispanics. ${ }^{12}$ All but two ${ }^{11} 12$ of these studies relied on self-reported race/ethnicity. Genetic ancestry has several advantages for defining 
ancestry compared with self-report, including greater objectivity and precision, particularly for persons with admixed backgrounds. Markers of genetic ancestry have been shown to improve accuracy in referencing lung function. ${ }^{13}$ We examined if the relationships of pack-years of smoking to lung function and per cent emphysema varied by genetic ancestry, continental ancestry or self-reported race/ethnicity in a large multi-ethnic cohort study.

\section{METHODS}

Multi-ethnic study of atherosclerosis

The Multi-Ethnic Study of Atherosclerosis (MESA) is a population-based prospective cohort that recruited 6814 participants ages 45-84 years in 2000-2002 from six US sites who were Caucasian, African-American, Hispanic or Asian (predominantly of Chinese origin). ${ }^{14}$ Each site recruited at least two race/ethnic groups and all race/ethnic groups were recruited at multiple sites. Exclusion criteria included clinical cardiovascular disease, pregnancy, weight $>300 \mathrm{lbs}$, inability to speak English, Spanish, Cantonese or Mandarin, and chest CT within the past year.

The MESA Family Study enrolled an additional 1612 African-American and Hispanic participants, predominantly siblings of MESA participants. The inclusion and exclusion criteria were identical, except that clinical cardiovascular disease was permitted.

The MESA Lung Study assessed per cent emphysema for all MESA and MESA Family participants and performed spirometry on a subset of participants. The subset was randomly sampled among those who consented to genetic analyses, underwent baseline measures of endothelial function, and attended an examination during the 2004-2006 MESA Lung recruitment period (figure 1). Chinese-Americans were oversampled to improve precision in this group. Participants with restrictive spirometry patterns, defined as a forced vital capacity (FVC) less than the lower limit of normal $(\mathrm{LLN})^{15}$ and a forced expiratory volume in $1 \mathrm{~s}\left(\mathrm{FEV}_{1}\right)$ to FVC ratio above the LLN, were excluded from analyses as the hypotheses relate specifically to obstructive lung disease.

The protocols of MESA, MESA Family and MESA Lung Studies were approved by the Institutional Review Boards of all collaborating institutions and the National Heart Lung Blood Institute.

\section{Genetic ancestry}

Genetic ancestry was defined using principal components $(\mathrm{PCs})^{16}$ derived from genome-wide data from the Affymetrix

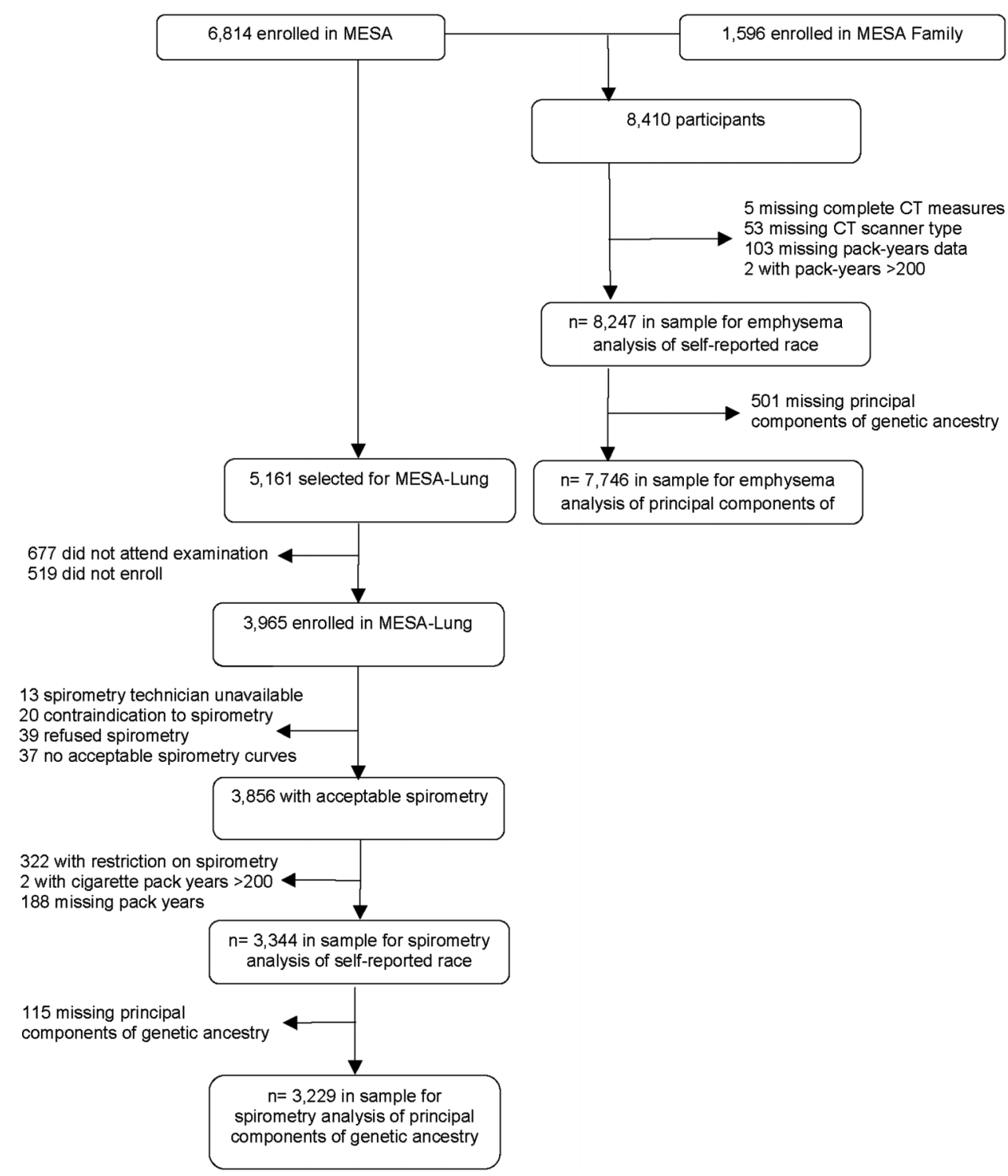

Figure 1 Participants in the Multi-Ethnic Study of Atherosclerosis (MESA) and MESA Family Studies in analyses for spirometry and emphysema. 

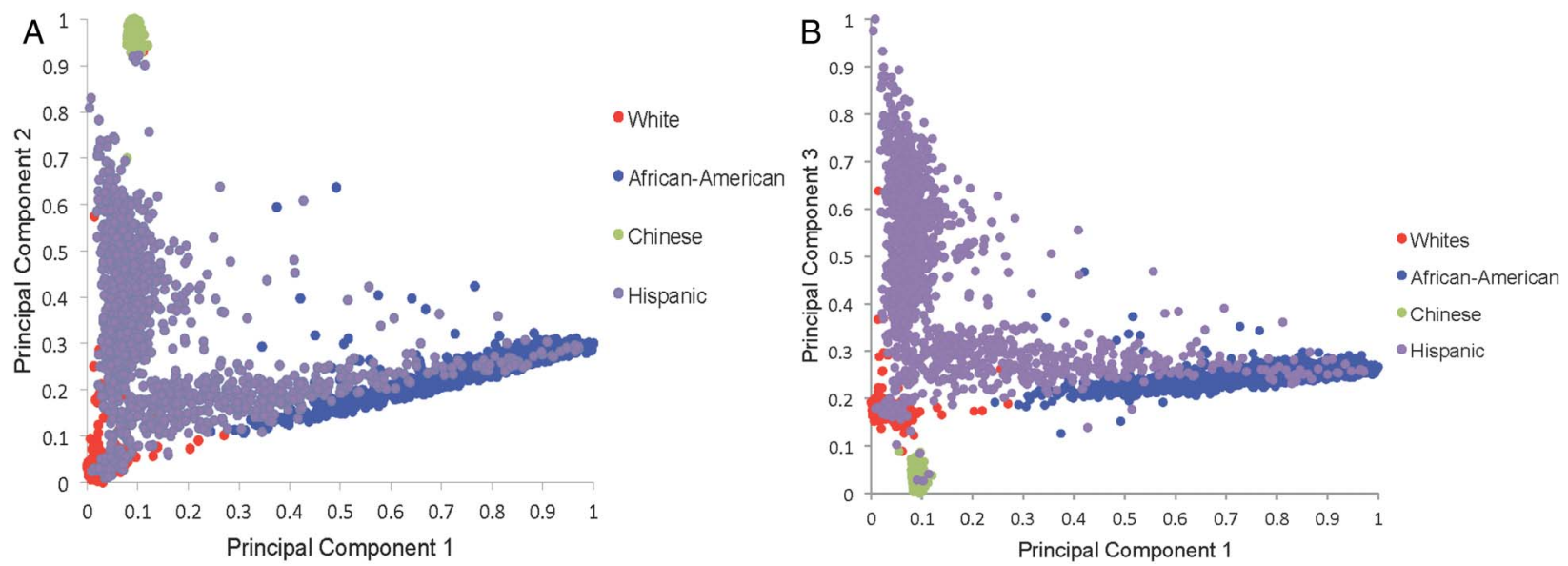

Figure 2 (A) Distribution of principal component 1 and principal component 2 by self-reported race. (B) Distribution of principal component 1 and principal component 3 by self-reported race.

6.0 chip among consenting participants available genetic data $(\mathrm{n}=8227)$. PC analysis, when applied to genotype data, allows for the transformation of a large number of correlated single nucleotide polymorphisms into a smaller number of continuous axes of variation that correspond to regions of geographic ancestry. ${ }^{16}$ A total of 50 PCs were defined. The first three PCs explain $86 \%$ of the total observed variation. A Cattell Scree plot $^{17}$ showed that the relative value of additional PCs beyond the third PC was very small. These three PCs also reveal three geographic clines: the first principal component (PC1) identifies variation between European and African ancestry; the second, PC2, identifies variation between European and Chinese ancestry (figure $2 \mathrm{~A}$ ); and the third, $\mathrm{PC} 3$, identifies variation across Hispanics (figure 2B).

Continental ancestry was assessed among African-Americans and Hispanics using ADMIXTURE. Among African-Americans, proportion of African ancestry was determined based on ADMIXTURE estimates from a two-way model. Among Hispanics, proportion of African and Native American ancestry was determined based on a three-population model.

\section{Race/ethnicity}

Race/ethnicity, age, gender, educational attainment and medical history were obtained via questionnaire. Categories for race/ethnicity were consistent with US 2000 Census definitions. ${ }^{18}$ Participants reported one of the following race/ethnicities: non-Hispanic white, African-American, Asian-American of Chinese descent or Hispanic/Latino. Participants who reported Hispanic ethnicity were classified as Hispanic regardless of selfreported race.

\section{Cumulative exposure to cigarette smoke (pack-years)}

Pack-years of cigarette smoking was calculated as: (years smoked) $\times($ cigarettes per day $/ 20)$ using standardised questionnaire items. ${ }^{19}$ Urinary cotinine level was assessed in the spirometry group by immunoassay (Immulite 2000 Nicotine Metabolite Assay; Diagnostic Products Corp., Los Angeles, California, USA). For self-reported former smokers whose cotinine levels were consistent with current smoking, years smoked was increased by a value equal to the time interval from last reported smoking to the time of cotinine assay.

Current smoking was defined as cigarette use in the last 30 days or a urinary cotinine level of $>100 \mathrm{ng} / \mathrm{ml}$.
Ever-smoking was defined as greater than 100 lifetime cigarettes smoked.

\section{Spirometry}

Spirometry was conducted in 2004-2006 in accordance with the American Thoracic Society/European Respiratory Society guidelines ${ }^{20}$ on a dry-rolling-sealed spirometer with automated quality checks (Occupational Marketing, Inc, Houston, Texas, USA). Spirometry exams were reviewed by one investigator. ${ }^{21}$ The intraclass correlation coefficient (ICC) of both $\mathrm{FEV}_{1}$ and FVC on random $10 \%$ replicate testing was 0.99 . Airflow obstruction was defined as $\mathrm{FEV}_{1}$ to FVC ratio below the LLN. ${ }^{22}$

\section{Per cent emphysema}

Emphysema was quantitatively measured on lung fields of cardiac CT scans obtained at full inspiration on multi-detector and electron-beam CT scanners, which included approximately $70 \%$ of the lung volume from the carina to the lung bases. ${ }^{23}$ Each participant underwent two scans and the scan with the greater volume of lung air was used, except in cases of discordant scan quality, when the higher-quality scan was analysed. ${ }^{24}$ Image attenuation was assessed with a modified version of the Pulmonary Analysis Software Suite ${ }^{25-27}$ at a single reading centre. As air outside the body has a mean attenuation of -1000 Hounsfield units, the attenuation of each pixel in the lung regions was corrected to equal measured pixel attenuation $\times(-1000 /$ mean air attenuation $)$. Per cent emphysema was defined as the percentage of the total voxels in the lung with attenuation of less than -910 Hounsfield units. Emphysema measurements from the cardiac scans correlated closely with those from full-lung scans in the same study participants. ${ }^{24}$ The inter-scan ICC of per cent emphysema on 100\% replicate scans was 0.94 .

\section{Additional variables}

MESA Lung participants were surveyed regarding factors relevant to lung disease including self-report of physician diagnosis before age 45 , hay-fever, family history of emphysema, occupational exposure to dust, fumes and smoke, and household environmental tobacco smoke exposure defined as living with a smoker. Cigar and pipe smoking was defined as previously described..$^{28}$ Depth of inhalation of cigarettes was assessed using standardised questionnaire items. ${ }^{29}$ Height was measured to the nearest $0.1 \mathrm{~cm}$ and weight was measured to the nearest pound. 


\section{Statistical analysis}

The cohort was stratified by race/ethnicity and gender for descriptive purposes. Analyses were stratified by gender, given gender differences in smoking history and lung function.

Initial multivariable regression models of lung function and airflow obstruction included age, age ${ }^{2}$, height ${ }^{2}$, pack-years, and either race/ethnicity or PCs of genetic ancestry, ${ }^{30}$ and adjusted for current smoking. Fully adjusted multivariable regression models additionally included body mass index, educational attainment, cigar smoking status, cigar pack-years, secondhand smoke exposure, depth of inhalation, time before first cigarette in the morning, urinary cotinine level, asthma, hay-fever, family history of emphysema, and occupational exposure to dust, fumes or smoke.

Linear regression was used for analyses of lung function and per cent emphysema and logistic regression for analyses of airflow obstruction. As the participants in analyses of per cent emphysema included related family members, these analyses employed generalised estimating equations ${ }^{31}$ to account for correlation between family members and were additionally adjusted for CT scanner type and dose. Participants with lung function measures did not include any related family members; thus, generalised estimating equations were not necessary.

Differences in the relationship between pack-years and lung function measures by genetic ancestry and race/ethnicity were tested in full multivariable models using the -2 log likelihood test of nested models with and without the interaction terms on an additive scale for lung function and lung density and a multiplicative scale for airflow obstruction. Sensitivity analyses were performed on the converse scales. As race and PCs of ancestry are collinear, they were not included in the same models; rather, two separate sets of analyses were performed. All models met the assumptions for linear and logistic regression, respectively. Presented results are untransformed. Statistical significance was defined as two-tailed $\mathrm{p}$ values $<0.05$. Analyses were performed using SAS V.9.2 (SAS Institute, Cary, North Carolina, USA).

\section{RESULTS}

Among 3344 participants in spirometry analyses using selfreported race, 35\% were non-Hispanic Caucasian, 26\% African-American, 22\% Hispanic and 17\% Chinese-American. The background of Hispanic participants was 51\% Mexican, 14\% Puerto Rican, 14\% Dominican, 4\% Cuban and 17\% other background. The mean age was 66 years; $48 \%$ were male subjects. In all, $11 \%$ were current smokers and $45 \%$ former smokers, with a median of 18 pack-years of cigarette smoking (IQR 6, 36) among ever-smokers.

Participant characteristics in the spirometry analysis are shown in table 1. Age and gender distributions were similar across race/ethnic groups. African-Americans were more likely to report current smoking than other groups. Pack-years of smoking were the greatest among Caucasians followed by African-Americans, Hispanics and Chinese-Americans. Women were less likely to have ever-smoked than men, and only 10 of 278 Chinese-American women reported ever-smoking.

Estimates of genetic ancestry were available for 3229 of the 3344 participants included in the spirometry analysis and followed the expected distribution (table 1).

\section{Cumulative smoking, genetic ancestry and lung function among men}

Pack-years were associated with significant decrements in lung function and increased ORs of airflow obstruction in all race/ ethnic groups. Among 1609 men, every 10 pack-years of smoking was associated with a mean decrement of $-0.69 \%$ $(95 \%$ CI $-0.92 \%$ to $-0.47 \%)$ in $\mathrm{FEV}_{1}$ to $\mathrm{FVC}$ ratio, a mean decrement of $-42.6 \mathrm{ml}(95 \% \mathrm{CI}-55.2$ to -30.0$)$ in $\mathrm{FEV}_{1}$ and a $1.14(95 \%$ CI 1.05 to 1.23$)$ increase in the odds of airflow obstruction.

There was no evidence that the relationship of pack-years to $\mathrm{FEV}_{1}$ to FVC ratio or airflow obstruction varied by genetic ancestry or self-reported race (table 2). Plots of the relationship of pack-years to $\mathrm{FEV}_{1}$ to FVC ratio showed linear, qualitatively similar relationships for all racial/ethnic groups (see online supplementary figure S1A). Findings were similar when performed on a multiplicative scale and when the outcome was per cent predicted $\mathrm{FEV}_{1}$ to $\mathrm{FVC}$ ratio (all $\mathrm{p}>0.1$ ).

The relationship of pack-years to $\mathrm{FEV}_{1}$, however, differed by genetic ancestry $(p=0.007)$ and self-reported race/ethnicity $(p=0.007) . P C 2$, which identifies differences in European and Asian ancestry, modified the effect of pack-years of smoking on $\mathrm{FEV}_{1}(\mathrm{p}=0.001)$ whereas interaction terms for pack-years of smoking with PC1 (European vs African ancestry) and PC3 (European vs Hispanic ancestry) were not statistically significant $(\mathrm{p}=0.30$ and 0.94$)$. Results for self-reported race were similar. When self-reported Chinese-American men were removed from the analysis, the interaction term no longer had a significant effect on $\mathrm{FEV}_{1}$ (genetic ancestry $\mathrm{p}=0.23$; self-reported race $\mathrm{p}=0.26$, table 2 parentheses).

The mean difference in the effect of 10 pack-years of smoking on $\mathrm{FEV}_{1}$ among African-Americans compared with non-Hispanic Caucasians was $7.0 \mathrm{ml}(95 \% \mathrm{CI}-18.5$ to 32.5$)$; the mean difference in the effect of 10 pack-years on $\mathrm{FEV}_{1}$ among Hispanics compared with Caucasians was $-0.6 \mathrm{ml}(95 \%$ CI -26.4 to 25.3). The mean difference in the effect of 10 pack-years on $\mathrm{FEV}_{1}$ among Chinese-Americans, however, was significantly different compared with non-Hispanic Caucasians, with a difference of $49.0 \mathrm{ml}(95 \%$ CI 18.8 to $79.3, \mathrm{p}=0.002)$. Evidence of an interaction between race/ethnicity and smoking on the $\mathrm{FEV}_{1}$ in men was also present on a multiplicative scale $(p=0.02$ for both genetic ancestry and self-reported race/ethnicity) and for per cent of predicted $\mathrm{FEV}_{1}(\mathrm{p}=0.02)$.

Among African-American men, there was no evidence for an interaction between proportion continental African ancestry and pack-years on $\mathrm{FEV}_{1}, \mathrm{FEV}_{1}$ to $\mathrm{FVC}$ ratio or per cent emphysema (all $\mathrm{p}>0.05$ ). In Hispanic-American male subjects, however, the interactions terms of pack-years with the $\mathrm{FEV}_{1}$ to $\mathrm{FVC}$ ratio were significant for Native American $(p=0.012)$ and African $(\mathrm{p}=0.030)$ ancestry (likelihood ratio test $\mathrm{p}=0.016)$, suggesting a lower $\mathrm{FEV}_{1}$ to $\mathrm{FVC}$ ratio with greater Native American and African ancestry. No such interaction was present for the $\mathrm{FEV}_{1}$.

\section{Genetic ancestry, cumulative smoking and lung function among women}

Among 1735 women, each 10 pack-years of smoking was associated with a $-0.85 \%(95 \% \mathrm{CI}-1.13 \%$ to $-0.57 \%)$ mean decrement in $\mathrm{FEV}_{1}$ to $\mathrm{FVC}$ ratio, a $-48.6 \mathrm{ml}(95 \% \mathrm{CI}-61.6$ to -35.7) mean decrement in $\mathrm{FEV}_{1}$ and a 1.36 (95\% CI 1.20 to $1.55)$ increase in the odds of airflow obstruction. Plots of the relationship of pack-years to $\mathrm{FEV}_{1}$ to $\mathrm{FVC}$ ratio showed similarly linear relationships for racial/ethnic groups (see online supplementary figure S1B).

There was no evidence that the relationship of pack-years to $\mathrm{FEV}_{1}, \mathrm{FEV}_{1}$ to $\mathrm{FVC}$ ratio or airflow obstruction differed by genetic ancestry among Caucasian, African-American and Hispanic women (table 3). Chinese-American women were excluded from this analysis given the very small number with a 
Table 1 Characteristics of the Multi-Ethnic Study of Atherosclerosis lung sample stratified by race/ethnicity and gender

\begin{tabular}{|c|c|c|c|c|c|c|c|c|}
\hline \multirow[b]{2}{*}{$n=3344$} & \multicolumn{4}{|l|}{ Men $(n=1609)$} & \multicolumn{4}{|l|}{ Women $(n=1735)$} \\
\hline & Non-Hispanic Caucasians & African-Americans & Hispanics & Chinese-Americans & Non-Hispanic Caucasians & African-Americans & Hispanics & Chinese-Americans \\
\hline$n(\%)$ & $582(36)$ & $402(25)$ & $342(21)$ & $283(18)$ & $591(34)$ & $471(27)$ & $395(23)$ & $278(16)$ \\
\hline Age, mean $(S D)$, years & $66(9.8)$ & $66(9.7)$ & $64(10.0)$ & $66(9.7)$ & $66(10.0)$ & $66(9.5)$ & $65(9.8)$ & $66(9.6)$ \\
\hline \multicolumn{9}{|l|}{ Smoking status, n (\%) } \\
\hline Never & 209 (36) & $129(32)$ & $124(36)$ & $142(50)$ & $288(49)$ & $252(54)$ & $269(68)$ & $268(96)$ \\
\hline Former & $328(56)$ & $198(49)$ & $175(51)$ & $119(42)$ & $255(43)$ & $160(34)$ & $102(26)$ & $6(2)$ \\
\hline Current & $45(8)$ & $75(19)$ & $43(13)$ & $22(8)$ & $48(8)$ & $59(13)$ & $24(6)$ & $4(1)$ \\
\hline Pack-years of smoking, ${ }^{*}$ median (IQR) & $24.0(9.0,44.0)$ & $20.3(8.8,36.8)$ & $16.3(5.9,34.5)$ & $17.5(5.4,33.0)$ & $18.9(6.0,36.8)$ & $17.3(6.4,33.0)$ & $7.0(2.0,16.5)$ & $13.5(2.0,19.5)$ \\
\hline \multicolumn{9}{|l|}{ Inhalational depth (cigarettes) † n (\%) } \\
\hline 'Shallow' & $25(7)$ & $12(4)$ & 17 (8) & 20 (14) & $11(4)$ & $16(7)$ & $22(17)$ & $2(20)$ \\
\hline 'Moderate' & $37(10)$ & $51(19)$ & $44(20)$ & $30(21)$ & $40(13)$ & $56(26)$ & $37(29)$ & $5(50)$ \\
\hline 'Deep' & $190(51)$ & $132(48)$ & $85(39)$ & $54(38)$ & $160(53)$ & $98(45)$ & $28(22)$ & $3(30)$ \\
\hline 'Very deep' & $105(28)$ & $60(22)$ & $49(22)$ & $25(18)$ & $67(22)$ & $38(17)$ & $23(18)$ & $0(0)$ \\
\hline Time until first cigaretteł (SD), h & $1.6(2.3)$ & $1.6(2.3)$ & $2.9(3.4)$ & $1.6(1.8)$ & $2.6(5.0)$ & $2.2(3.1)$ & $3.5(3.9)$ & $6.0(6.4)$ \\
\hline Cigar use* $\mathrm{n}(\%)$ & $155(42)$ & $67(25)$ & $36(17)$ & $6(5)$ & $10(3)$ & $10(5)$ & $2(2)$ & $0(0)$ \\
\hline Cigar years,§ median (IQR) & $20(10,39)$ & $15(7,55)$ & $10(6,24)$ & $29(16,51)$ & $16(8,28)$ & $17(5,30)$ & $0(0,0)$ & $0(0,0)$ \\
\hline Height, mean (SD), cm & $176(6.9)$ & $176(6.8)$ & $169(6.4)$ & $168(6.4)$ & $162(6.6)$ & $162(6.9)$ & $155(5.8)$ & $155(6.1)$ \\
\hline BMI, mean (SD), kg/m² & $28(4.1)$ & $28(4.6)$ & $29(4.1)$ & $24(3.2)$ & $28(5.8)$ & $31(6.2)$ & $30(5.4)$ & $24(3.4)$ \\
\hline \multicolumn{9}{|l|}{ Educational attainment n (\%) } \\
\hline High school or vocational school & $108(19)$ & $134(33)$ & 190 (56) & $69(24)$ & $134(23)$ & $138(29)$ & $235(59)$ & $94(34)$ \\
\hline Incomplete college & $104(18)$ & $135(34)$ & $108(32)$ & $60(21)$ & $181(31)$ & $157(33)$ & $131(33)$ & $99(36)$ \\
\hline Complete college & $149(26)$ & 70 (17) & $24(7)$ & $76(27)$ & $138(23)$ & $90(19)$ & $14(4)$ & $59(21)$ \\
\hline Graduate or professional school & $221(38)$ & $63(16)$ & $20(6)$ & $78(28)$ & $138(23)$ & $86(18)$ & $15(4)$ & $26(9)$ \\
\hline Household ETS exposure $\mathrm{n}(\%)$ & $229(39)$ & $176(44)$ & $93(27)$ & $52(18)$ & $310(52)$ & $284(60)$ & $173(44)$ & $119(43)$ \\
\hline Occupational exposureף n (\%) & $269(46)$ & $237(59)$ & $237(59)$ & $62(22)$ & $213(36)$ & $221(47)$ & $177(45)$ & $44(16)$ \\
\hline Asthma** $\mathrm{n}(\%)$ & $52(9)$ & $32(8)$ & $16(5)$ & $14(5)$ & $54(9)$ & $56(11)$ & $38(10)$ & $10(4)$ \\
\hline Hay-fevertt n (\%) & $200(34)$ & $114(28)$ & $70(20)$ & $90(32)$ & $238(40)$ & $189(40)$ & $112(28)$ & $88(32)$ \\
\hline \multicolumn{9}{|c|}{ Ancestral principal components (PC), median, (IQR) (n=3229) } \\
\hline PC1 & $0.020(0.015,0.043)$ & $0.805(0.698,0.870)$ & $0.073(0.054,0.169)$ & $0.091(0.088,0.095)$ & $0.0199(0.015,0.036)$ & $0.804(0.689,0.876)$ & $0.089(0.062,0.200)$ & $0.091(0.088,0.095)$ \\
\hline PC2 & $0.032(0.026,0.043)$ & $0.252(0.222,0.272)$ & $0.307(0.189,0.425)$ & $0.977(0.971,0.982)$ & $0.032(0.025,0.042)$ & $0.252(0.222,0.274)$ & $0.299(0.202,0.432)$ & $0.976(0.969,0.982)$ \\
\hline PC3 & $0.175(0.167,0.182)$ & $0.250(0.240,0.259)$ & $0.451(0.284,0.564)$ & $0.027(0.019,0.038)$ & $0.176(0.170,0.184)$ & $0.250(0.240,0.256)$ & $0.428(0.294,0.566)$ & $0.028(0.019,0.040)$ \\
\hline \multicolumn{9}{|l|}{ Lung function } \\
\hline $\mathrm{FEV}_{1}(\mathrm{SD}), \mathrm{L}$ & $3.0(0.7)$ & $2.6(0.6)$ & $3.0(0.7)$ & $2.6(0.6)$ & $2.2(0.5)$ & $1.9(0.4)$ & $2.0(0.5)$ & $1.8(0.4)$ \\
\hline FVC (SD), L & $4.3(0.8)$ & $3.6(0.7)$ & $3.9(0.8)$ & $3.5(0.7)$ & $2.9(0.6)$ & $2.4(0.5)$ & $2.6(0.5)$ & $2.4(0.5)$ \\
\hline $\mathrm{FEV}_{1}$ to FVC ratio (SD), \% & $72(9.1)$ & $72(10.7)$ & $75(8.8)$ & $74(8.6)$ & $74(7.8)$ & $77(8.0)$ & $78(6.9)$ & $76(6.6)$ \\
\hline 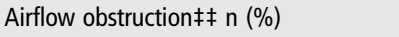 & $79(21)$ & $63(23)$ & $31(14)$ & $9(6)$ & $57(19)$ & $23(11)$ & $11(9)$ & $1(10)$ \\
\hline
\end{tabular}




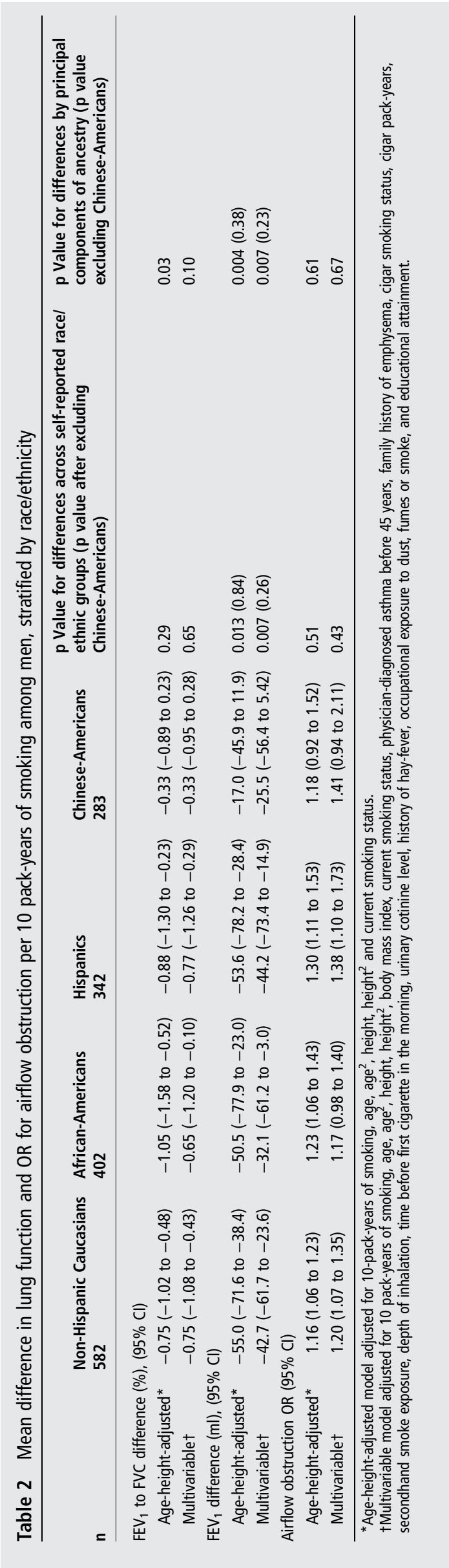

smoking history. Similarly, there was also no evidence for effect modification by self-reported race/ethnicity among women (table 3).

Among African-American and Hispanic women, there was no evidence for any interaction between pack-years and proportion African ancestry and, among Hispanics, Native American ancestry for the $\mathrm{FEV}_{1}$ or $\mathrm{FEV}_{1}$ to FVC ratio (all $\mathrm{p}>0.05$ ).

\section{Cumulative smoking and per cent emphysema}

Characteristics of 8247 participants included in the analyses of per cent emphysema are shown in online supplementary table S1. Among women, every 10 pack-years of smoking were associated with a $0.43 \%$ increase in per cent emphysema $(p<0.001)$. Among men, 10 pack-years of smoking was associated with a $0.10 \%$ increase in per cent emphysema, though the association was not statistically significant $(p=0.30)$. There was no evidence that this association differed by genetic ancestry among men or women, although in women there was a suggestion of effect modification by self-reported race/ethnicity $(\mathrm{p}=0.03$; online supplementary table S2). Furthermore, there was no evidence that the association of pack-years to per cent emphysema varied by continental ancestry among African-American and Hispanic women and men $(\mathrm{p}>0.16)$.

\section{Sensitivity analyses}

Among 1255 men and women with a history of smoking greater than 10 pack-years (mean pack-years $36, \mathrm{SD} \pm 26$ ), there was also no evidence of that the relationship of pack-years to $\mathrm{FEV}_{1}, \mathrm{FEV}_{1}$ to FVC ratio or airflow obstruction differed by self-reported race/ ethnicity or genetic ancestry (see online supplementary table S3).

In the present study sample, $96 \%$ of Chinese, $69 \%$ of Hispanics, $9 \%$ of African-Americans and 7\% of Caucasians were immigrants to the USA. Among Hispanics (as well as Caucasians and African-Americans), there was no evidence that either immigrant status or years lived outside the USA was associated with the $\mathrm{FEV}_{1}$ or $\mathrm{FEV}_{1}$ to $\mathrm{FVC}$ ratio. Among Chinese-Americans, immigrant status was not associated with either $\mathrm{FEV}_{1}$ or $\mathrm{FEV}_{1}$ to $\mathrm{FVC}$ ratio $(p=0.37$ and $p=0.72$, respectively). Years lived outside the USA was not associated with $\mathrm{FEV}_{1}(\mathrm{p}=0.19)$ but was associated with a small decrement in mean $\mathrm{FEV}_{1}$ to $\mathrm{FVC}$ ratio $(-0.06 \%,(95 \% \mathrm{CI}$ $-0.11 \%$ to $-0.02 \%) \mathrm{p}=0.001)$; additional adjustment for immigrant status and years lived outside the USA did not, however, alter the main results on the relationship among race, pack-years and lung function (see online supplementary table S4).

Since MESA excluded participants with clinical cardiovascular disease, we repeated analyses restricted to participants aged 4564 years, an age range in which clinical cardiovascular disease is rare, and found similar results (see online supplementary table S5). Site-specific analyses demonstrated no significant interactions with the $\mathrm{FEV}_{1}$ to $\mathrm{FVC}$ ratio and a significant interaction with the $\mathrm{FEV}_{1}$ at one of the two sites that recruited Chinese-Americans (see online supplementary table S6), although the direction of association was inconsistent across the six sites.

\section{DISCUSSION}

In this large, population-based sample, there was no consistent evidence that the associations of cumulative smoking with $\mathrm{FEV}_{1}$ to FVC ratio, airflow obstruction or per cent emphysema varied by genetic ancestry among the four largest race/ethnic groups in the USA.

Two recent studies have used ancestral informative markers (AIMs) to assess for interaction between genetic ancestry and smoking. A case-control study by Bruse et $a l^{12}$ of variation in tobacco-related susceptibility to COPD by genetic ancestry found that Hispanic smokers had lower odds of COPD and reduced 
Table 3 Mean difference in lung function and OR for airflow obstruction per 10 pack-years of smoking among women, stratified by race/ ethnicity

\begin{tabular}{|c|c|c|c|c|c|c|}
\hline n. & $\begin{array}{l}\text { Non-Hispanic } \\
\text { Caucasians } \\
591\end{array}$ & $\begin{array}{l}\text { African-Americans } \\
471\end{array}$ & $\begin{array}{l}\text { Hispanics } \\
395\end{array}$ & $\begin{array}{l}\text { Chinese-Americans }{ }^{*} \\
278\end{array}$ & $\begin{array}{l}\mathrm{p} \text { Value for } \\
\text { differences across } \\
\text { race/ethnic groups }\end{array}$ & $\begin{array}{l}p \text { Value for } \\
\text { differences } \\
\text { by principal } \\
\text { components of } \\
\text { ancestry }\end{array}$ \\
\hline \multicolumn{7}{|c|}{$\mathrm{FEV}_{1}$ to $\mathrm{FVC}$ difference $(\%),(95 \% \mathrm{Cl})$} \\
\hline \multicolumn{6}{|c|}{ Age-height-adjusted* ${ }^{*}$} & 0.29 \\
\hline Multivariablet & $-0.97(-1.38$ to -0.56$)$ & $-0.82(-1.35$ to -0.29$)$ & $-0.65(-1.30$ to 0.01$)$ & & 0.29 & 0.32 \\
\hline \multicolumn{7}{|c|}{$\mathrm{FEV}_{1}$, mean difference $(\mathrm{ml}),(95 \% \mathrm{Cl})$} \\
\hline \multicolumn{6}{|c|}{ 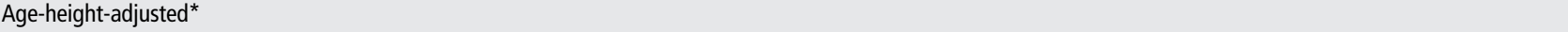 } & 0.99 \\
\hline Multivariablet & $-41.1(-59.8$ to -22.5$)$ & $-47.1(-70.5$ to -23.8$)$ & $-36.7(-69.0$ to -4.5$)$ & & 0.43 & 0.25 \\
\hline \multicolumn{7}{|c|}{ Airflow obstruction OR $(95 \% \mathrm{Cl})$} \\
\hline \multicolumn{7}{|c|}{ Age-height-adjusted* } \\
\hline Multivariablet & 1.29 (1.08 to 1.54$)$ & 1.49 (1.16 to 1.54$)$ & 1.53 (0.85 to 2.74$)$ & & 0.25 & 0.83 \\
\hline
\end{tabular}

decline in $\mathrm{FEV}_{1}$ compared with non-Hispanic Caucasians with an equal cumulative smoking history. These findings were not replicated in our present study; however, differences between the studies include the use of AIMs in the former case-control study compared with PCs based upon 1 million single nucleotide polymorphisms in the present population-based study. Additionally, as the study by Bruse et al recruited Hispanics at one site (Albuquerque, New Mexico, USA), predominantly of regional Native American and Mexican origin, its findings might apply specifically to Hispanics of the Southwestern region of the USA while the present multicentre study recruited Hispanics of both Mexican and Caribbean origin. Also, differences in mean packyears may have contributed to variation in results. The mean pack-years in the present study is 16 among non-Hispanic Caucasians and 10 among Hispanics where mean pack-years in the aforementioned study was higher ( 34 for Hispanics and 41 for non-Hispanic Caucasians).

We also found no evidence of a higher risk of COPD among African-Americans in contrast to a case-control study of 70 cases of early-onset COPD, ${ }^{8}$ a retrospective review of 160 patients presenting for lung volume reduction surgery, ${ }^{9}$ and a prospective study of 50 African-Americans and 278 Caucasians, ${ }^{10}$ all using self-reported race/ethnicity. One explanation for these differences is that prior findings in early-onset and very severe COPD may not apply to the general population and, conversely, findings in the general population may not apply to these extreme phenotypes. Alternatively, small, case-control studies may be subject to selection bias. Notably, a more recent study incorporating genetic measures by Aldrich et $a l^{11}$ used AIMs and identified a trend, though non-significant, toward an interaction between African ancestry and smoking on $\mathrm{FEV}_{1}$ in cross-sectional and longitudinal analysis among self-reported African-Americans. These findings were not replicated in our present study. Differences include an older cohort with a higher mean pack-years (30) among the participants in the study by Aldrich et al as well as the longitudinal approach, suggesting that it could be possible that there is more variability by race as individuals age. Our results are, however, consistent with a large meta-analysis of population-based studies using self-reported race-ethnicity. ${ }^{7}$

The present study was unique in enrolling Chinese-Americans along with the three other race/ethnic groups in the same study. We found no evidence of a differential risk in this group for $\mathrm{FEV}_{1}$ to $\mathrm{FVC}$ ratio, airflow limitation and per cent emphysema; however, the association between cumulative smoking and $\mathrm{FEV}_{1}$ was modified by genetic ancestry among men of Chinese-American ancestry. These results build on findings from the prior meta-analysis of lung function, which found that selfreported Asian/Pacific Islanders had smaller smoking-related decrements in $\mathrm{FEV}_{1}$ than Caucasians. ${ }^{7}$ The specificity of the interaction in $\mathrm{FEV}_{1}$ suggests that it may be related to mean differences in body size among Asian men compared with other race/ethnic groups that are not fully indexed by height. ${ }^{21}$ Other possible explanations for this difference include dietary and lifestyle factors. For example, mean levels of n-3 polyunsaturated fatty acids are substantially higher among Asians and Caucasians compared with other groups in MESA, ${ }^{32}$ which may contribute to a lower risk of COPD. ${ }^{33}$

Among women, but not men, we identified a statistically significant effect modification on per cent emphysema by selfreported race $(p=0.03)$, and a trend toward effect modification by ancestry $(p=0.10$; see online supplementary table $S 2)$. One potential explanation for this finding is a sex-specific locus that determines smoking-related emphysema changes, which may provide an interesting avenue for future research.

Overall, these findings suggest that the effect of cumulative smoking on COPD does not vary substantially among the four major race/ethnic groups in the USA. Observed race/ethnic disparities in COPD in the USA may instead result from differences in smoking patterns, differential exposure to air pollution or environmental toxins, maternal smoking during pregnancy, ${ }^{34}$ low birth weight, ${ }^{35}$ exposure to pulmonary irritants during lung 
development ${ }^{9}$ and occupational exposures. Different smoking habits and brands of cigarettes have also been cited, although depth of inhalation was similar across race/ethnic groups in this study.

This study has a number of strengths, including advanced assessment of genetic ancestry, a population-based study which avoids site-by-race confounding and limits selection bias, large sample size and standardised methods.

Smoking history may be subject to inaccurate reporting; however, results would only be biased if misclassification of pack-years were differential by race/ethnicity. Current smoking was confirmed with cotinine levels in MESA Lung participants, and the accuracy of self-reported current smoking did not differ by race/ethnicity $(p=0.34)$. Cigarette brand and type was not assessed; however, COPD risk does not vary substantially by brand or type. ${ }^{36}$

Use of genetic PCs of ancestry may carry biases. If, for example, we seek to control for cultural confounders such as dietary and environmental factors that may be associated with race/ethnic group, using genetic ancestry may potentially misclassify persons who culturally identify with one group while genetic ancestry is admixed.

Additionally, genetic PCs of ancestry as used in these analyses may not capture within group variation particularly among the highly admixed African-American and Hispanic groups. In order to address this issue, we performed analyses using individual continental ancestry proportions. Among African-Americans, we again found no interaction between continental ancestry and pack-years of smoking on lung function. Among Hispanic-American male subjects we found a statistically significant increase in the effect of pack-years on $\mathrm{FEV}_{1}$ to $\mathrm{FVC}$ ratio proportion of Native-American and African ancestry. This finding is in contrast to the finding of a prior study. ${ }^{11}$ One potential explanation for this difference is that the current study recruited Hispanics from multiple geographic regions across the USA unlike the former study which recruited a population from one site in New Mexico, while it is also possible that either or both findings could be false positives given that there were multiple comparisons performed.

Postbronchodilator spirometry, used to define COPD, was not available in this cohort; however, epidemiological and genetic risk factors for an obstructive pattern of spirometry are similar to those for COPD ${ }^{37}$ and we used a contemporary definition of airflow limitation. Emphysema was assessed on partial lung scans. Although we have previously validated per cent emphysema measures from these scans compared with full-lung scans in MESA (ICC 0.94), ${ }^{37}$ the lung apices were not included in the partial lung scans that resulted in less precise effect estimates for smoking-related emphysema, which has an apical predilection. ${ }^{38}$ Nonetheless, the variability of these partial lung scans was comparable with that defined by full lung scans in other cohort studies. ${ }^{39}$ It should also be noted that results from this crosssectional study may not necessarily apply to longitudinal change in lung function and per cent emphysema.

Although MESA is a population-based study, participants with clinical cardiovascular disease were excluded; hence, the average smoking history was slightly less than the US population. ${ }^{40}$ The results, then, may not be fully generalisable to populations with heavier smoking exposures and very severe COPD. Last, though this study assessed PCs of ancestry which map along global ancestral clines, it is possible studies of locus-specific ancestry might yield different findings.

In conclusion, there was no strong evidence that the association of cigarette smoking to airflow limitation and emphysema varied by genetic ancestry in the four main US race/ethnic groups. Risks of smoking appear equally shared across the population.

\section{Author affiliations}

${ }^{1}$ Department of Medicine, Columbia University Medical Center, New York,

New York, USA

${ }^{2}$ Department of Biostatistical Sciences, Division of Public Health Sciences, Wake

Forest University Health Sciences, Winston-Salem, North Carolina, USA

${ }^{3}$ Center for Public Health Genomics, University of Virginia, Charlottesville, Virginia,

USA and Division of Biostatistics and Epidemiology, Department of Public Health

Sciences, University of Virginia, Charlottesville, Virginia, USA

${ }^{4}$ Department of Radiology, Division of Radiological Sciences, Wake-Forest University,

Winston-Salem, North Carolina, USA

${ }^{5}$ Department of Radiology, Los Angeles Biomedical Research Institute at Harbor-

UCLA Medical Center, Torrance, California, USA

${ }^{6}$ Department of Radiology, University of lowa, lowa City, lowa, USA

${ }^{7}$ Department of Biostatistics, University of Washington, Seattle, Washington, USA

${ }^{8}$ Department of Preventative Medicine, Northwestern University Medical School,

Chicago, Illinois, USA

${ }^{9}$ Department of Medicine, Johns Hopkins University, Baltimore, Maryland, USA

${ }^{10}$ Division of Epidemiology and Biostatistics, University of Arizona, Tucson, Arizona, USA

${ }^{11}$ Department of Medicine, University of California at Los Angeles, Los Angeles,

California, USA

${ }^{12}$ Medical Genetics Institute, Cedars-Sinai Medical Center, Los Angeles, California, USA

${ }^{13}$ Department of Epidemiology, Columbia University Medical Center, New York, New York, USA

Acknowledgements We acknowledge all participants and collaborators who contributed to the MESA and MESA-Family studies.

Contributors Primary data analysis and manuscript writing and preparation were performed by RP and DD. Additional analysis performed by AM. Manuscript review and editing recommendations were made by all authors. Data collection was performed by JD, JJC, RD, EAH, RAK, KL, RJ, NMP, ES, KEW, JIR, KDT, SSR and RGB. Funding was obtained by RGB, SSR and JIR. RGB provided senior author supervision.

Funding The MESA and MESA Lung Studies are conducted and supported by the NHLBI (contracts N01-HC-95159 through N01-HC-95165 and N01-HC-95169 and grants R01 HL-077612, R01 HL-075476 and RC1-HL100543) in collaboration with the MESA and MESA-Lung Investigators. This manuscript has been reviewed by the MESA. Investigators for scientific content and consistency of data interpretation with previous MESA publications and significant comments have been incorporated prior to submission for publication. The authors thank the other investigators, staff and participants of the MESA and MESA-Lung Studies for their valuable contributions. A full list of participating MESA Investigators and institutions can be found online (http://www.mesa-nhlbi.org).

Competing interests EAH is a founder and share holder of Vida Diagnostics. Data analysis used software from VIDA. RGB received costs of conference travel from Boeringher Ingelheim.

Patient consent Obtained.

Ethics approval IRB from all participating universities and the National Heart Lung Blood Institute (NHLBI).

Provenance and peer review Not commissioned; internally peer reviewed.

Data sharing statement Researchers interested in working with MESA Investigators are welcome to submit a manuscript proposal or ancillary study proposal directly to the study. Please feel free to review additional materials related to establishing a collaborative relationship with MESA at the following link http://www.mesa-nhlbi.org/ancillary.aspx

\section{REFERENCES}

1 Celli BR, MacNee W. Standards for the diagnosis and treatment of patients with COPD: a summary of the ATS/ERS position paper. Eur Respir J 2004;23:932-46.

2 Murray CJ, Lopez AD. Alternative projections of mortality and disability by cause 1990-2020: Global Burden of Disease Study. Lancet 1997;349:1498-504.

3 Pauwels RA, Buist AS, Calverley PMA, et al. Global strategy for the diagnosis, management, and prevention of chronic obstructive pulmonary disease. NHLBI/WHO Global Initiative for Chronic Obstructive Lung Disease (GOLD) Workshop Summary. Am J Respir Crit Care Med 2001;163:1256-76.

4 Rabe KF, Hurd S, Anzueto A, et al. Global strategy for the diagnosis, management, and prevention of chronic obstructive pulmonary disease: GOLD executive summary. Am J Respir Crit Care Med 2007;176:532-55. 
5 Mannino DM, Homa D, Akinbami $\sqcup$, et al. Chronic obstructive pulmonary disease surveilance-United States, 1971-2000. Surveillance Summaries MMWR 2002:511-8.

6 Akinbami LJ LX. Chronic obstructive pulmonary disease among adults aged 18 and over in the United States, 1998-2009. NCHS Data Brief, no 63. Hyattsville, MD: National Center for Health Statistics, 2011.

7 Vollmer WM, Enright PL, Pedula KL, et al. Race and gender differences in the effects of smoking on lung function. Chest 2000;117:764-72.

8 Foreman MG, Zhang L, Murphy J, et al. Early-onset chronic obstructive pulmonary disease is associated with female xex, maternal factors, and African American race in the COPDGene study. Am J Respir Crit Care Med 2011;184:414-20.

9 Chatila WM, Wynkoop WA, Vance G, et al. Smoking patterns in African Americans and whites with advanced COPD. Chest 2004;125:15-21.

10 Dransfield MT, Davis JJ, Gerald LB, et al. Racial and gender differences in susceptibility to tobacco smoke among patients with chronic obstructive pulmonary disease. Respir Med 2006;100:1110-16.

11 Aldrich MC, Kumar R, Colangelo LA, et al. Genetic ancestry-smoking interactions and lung function in African Americans: a cohort study. PLOS ONE 2012;7:e39541.

12 Bruse S, Sood A, Petersen $H$, et al. New Mexican Hispanic smokers have lower odds of chronic obstructive pulmonary disease and less decline in lung function than non-Hispanic Whites. Am J Respir Crit Care Med 2011;184:1254-60.

13 Kumar R, Seibold MA, Aldrich MC, et al. Genetic ancestry in lung-function predictions. N Engl J Med 2010;363:321-30.

14 Bild DE BD, Burke GL, Detrano R, et al. Multi-ethnic study of atherosclerosis: objectives and design. Am J Epidemiol 2002;156:871-81.

15 Hankinson JL, Odencrantz JR, Fedan KB. Spirometric reference values from a sample of the general U.S. population. Am J Respir Crit Care Med 1999;159:179-87.

16 Price AL, Patterson NJ, Plenge RM, et al. Principal components analysis corrects for stratification in genome-wide association studies. Nat Genet 2006;38:904-9.

17 Coste J, Bouee S, Ecosse E, et al. Methodological issues in determining the dimensionality of composite health measures using principal component analysis: case illustration and suggestions for practice. Qual Life Res 2005;14:641-54.

18 US Census Bureau. Overview of Race and Hispanic Origin Census 2000 Brief, March 2001.

19 Ferris BG. Epidemiology standardization project. Am Rev Respir Dis 1978;118 (Suppl 2):1-120.

20 Pellegrino R, Viegi G, Brusasco V, et al. Interpretative strategies for lung function tests. Eur Respir J 2005:26:948-68.

21 Hankinson JL, Kawut SM, Shahar E, et al. Performance of American Thoracic Society-recommended spirometry reference values in a multiethnic sample of adults: the multi-ethnic study of atherosclerosis (MESA) lung study. Chest 2010;137:138-45.

22 Vollmer WM, Gislason T, Burney $\mathrm{P}$, et al. Comparison of spirometry criteria for the diagnosis of COPD: results from the BOLD study. Eur Respir J 2009:34:588-97.

23 Carr JJ, Nelson JC, Wong ND, et al. Calcified coronary artery plaque measurement with cardiac CT in population-based studies: standardized protocol of Multi-Ethnic Study of Atherosclerosis (MESA) and Coronary Artery Risk Development in Young Adults (CARDIA) study. Radiology 2005;234:35-43.
24 Hoffman EA, Jiang $\mathrm{R}$, Baumhauer $\mathrm{H}$, et al. Reproducibility and validity of lung density measures from cardiac CT Scans-The Multi-Ethnic Study of Atherosclerosis (MESA) Lung Study. Acad Radiol 2009;16:689-99.

25 Tschirren J, McLennan G, Palagyi K, et al. Matching and anatomical labeling of human airway tree. IEEE Trans Med Imaging 2005;24:1540-7.

26 Hu S, Hoffman EA, Reinhardt JM. Automatic lung segmentation for accurate quantitation of volumetric $\mathrm{X}$-ray $\mathrm{CT}$ images. IEEE Trans Med Imaging 2001;20:490-8.

27 Zhang L, Hoffman EA, Reinhardt JM. Atlas-driven lung lobe segmentation in volumetric X-ray CT images. IEEE Trans Med Imaging 2006;25:1-16.

28 Rodriguez J, Jiang R, Johnson WC, et al. The association of pipe and cigar use with cotinine levels, lung function, and airflow obstruction: a cross-sectional study. Ann Intern Med 2010;152:201-10.

29 Heatherton TF, Kozlowski LT, Frecker RC, et al. The Fagerstrom Test for Nicotine Dependence: a revision of the Fagerstrom Tolerance Questionnaire. $\mathrm{Br}$ J Addict 1991;86:1119-27.

30 Kiefer EM, Hankinson JL, Barr RG. Similar relation of age and height to lung function among Whites, African Americans, and Hispanics. Am J Epidemiol 2011;173:376-87.

31 Zeger SL, Liang KY. Longitudinal data analysis for discrete and continuous outcomes. Biometrics 1986:42:121-30.

32 Anderson JS, Nettleton JA, Herrington DM, et al. Relation of omega-3 fatty acid and dietary fish intake with brachial artery flow-mediated vasodilation in the Multi-Ethnic Study of Atherosclerosis. Am J Clin Nutr 2010;92:1204-13.

33 Shahar E, Folsom AR, Melnick SL, et al. Dietary n-3 polyunsaturated acids and smoking-related chronic obstructive pulmonary disease. Am J Epidemiol 2008;168:796-801.

34 Gilliland FD, Berhane K, McConnell R, et al. Maternal smoking during pregnancy, environmental tobacco smoke exposure and childhood lung function. Thorax 2000:55:271-6

35 Barker DJ, Godfrey KM, Fall C, et al. Relation of birth weight and childhood respiratory infection to adult lung function and death from chronic obstructive airways disease. BMJ 1991;303:671-5.

36 Murray RP, Connett JE, Skeans MA, et al. Menthol cigarettes and health risks in Lung Health Study data. Nicotine Tob Res 2007;9:101-7.

37 Gibson PG, Simpson JL. The overlap syndrome of asthma and COPD: what are its features and how important is it? Thorax 2009;64:728-35.

38 Hogg JC. Pathophysiology of airflow limitation in chronic obstructive pulmonary disease. Lancet 2004;364:709-21.

39 Gietema HA, Schilham AM, van Ginneken B, et al. Monitoring of smoking-induced emphysema with $\mathrm{CT}$ in a lung cancer screening setting: detection of real increase in extent of emphysema. Radiology 2007;244:890-7.

40 Centers for Disease Control and Prevention (ed.) Vital signs: current cigarette smoking among adults aged $>$ or $=18$ years-United States, 2009. , MMWR 2010:59:1135-40. 